\title{
Article
}

\section{FinnTwin16: A Longitudinal Study from Age 16 of a Population-Based Finnish Twin Cohort}

\author{
Milla Kaidesoja ${ }^{1,2}$, Sari Aaltonen², Leonie H. Bogl ${ }^{2,3}$, Kauko Heikkilä², Sara Kaartinen ${ }^{1}$, Urho M. Kujala ${ }^{4}$, Ulla Kärkkäinen ${ }^{1}$, \\ Guiomar Masip ${ }^{1}$, Linda Mustelin ${ }^{1,2,5}$, Teemu Palviainen², Kirsi H. Pietiläinen ${ }^{6,7}$, Mirva Rottensteiner ${ }^{4,8}$, Pyry N. Sipilä1,9, \\ Richard J. Rose ${ }^{10}$, Anna Keski-Rahkonen ${ }^{1, \star}$ and Jaakko Kaprio ${ }^{1,2, \star}$ \\ ${ }^{1}$ Department of Public Health, University of Helsinki, Helsinki, Finland, ${ }^{2}$ Institute for Molecular Medicine FIMM, University of Helsinki, Helsinki, Finland, \\ ${ }^{3}$ Department of Epidemiology, Center for Public Health, Medical University of Vienna, Vienna, Austria, ${ }^{4}$ Faculty of Sport and Health Sciences, University of \\ Jyväskylä, Jyväskylä, Finland, ${ }^{5}$ Nightingale Health Ltd., Helsinki, Finland, ${ }^{6}$ Obesity Research Unit, Research Program for Clinical and Molecular Metabolism, \\ Faculty of Medicine, University of Helsinki, Helsinki, Finland, ${ }^{7}$ Obesity Center, Endocrinology, Abdominal Center, Helsinki University Central Hospital and \\ University of Helsinki, Helsinki, Finland, ${ }^{8}$ Department of Medicine, Central Finland Health Care District, Jyväskylä, Finland, ${ }^{9}$ Helsinki Institute of Life Science, \\ University of Helsinki, Helsinki, Finland and ${ }^{10}$ Department of Psychological \& Brain Sciences, Indiana University, Bloomington, IN, USA
}

\begin{abstract}
The purpose of this review is to provide a detailed and updated description of the FinnTwin16 (FT16) study and its future directions. The Finnish Twin Cohort comprises three different cohorts: the Older Twin Cohort established in the 1970s and the FinnTwin12 and FT16 initiated in the 1990s. FT16 was initiated in 1991 to identify the genetic and environmental precursors of alcoholism, but later the scope of the project expanded to studying the determinants of various health-related behaviors and diseases in different stages of life. The main areas addressed are alcohol use and its consequences, smoking, physical activity, overall physical health, eating behaviors and eating disorders, weight development, obesity, life satisfaction and personality. To date, five waves of data collection have been completed and the sixth is now planned. Data from the FT16 cohort have contributed to several hundred studies and many substudies, with more detailed phenotyping and collection of omics data completed or underway. FT16 has also contributed to many national and international collaborations.
\end{abstract}

Keywords: Alcohol; diet; diverse phenotypes; genetics; longitudinal twin-family study; mental health; obesity; physical activity; smoking; twins; weight

(Received 18 June 2019; accepted 7 August 2019; First Published online 3 December 2019)

During the past 30 years, the FinnTwin16 (FT16) study has created a rich longitudinal dataset of a well-characterized population-based sample of twins. In addition to providing valuable longitudinal data on twin pairs, FT16 offers data on population level because it is register-based with high participation rates yielding epidemiological data on a well-defined demographic group in Finland.

The National Institute on Alcohol Abuse and Alcoholism (of the US National Institutes of Health) provided funding for the initiation of the FT16 study in the 1990s. The original aim was to identify genetic and familial, and extrafamilial environmental precursors of alcoholism. The FT16 dataset has offered valuable information on different factors related to alcohol use/abuse and has expanded to include several different research areas linked to health-related behaviors and disease. In the five rounds of data collection executed thus far, the most prominent research areas in

Author for correspondence: Jaakko Kaprio, Email: jaakko.kaprio@helsinki.fi *Shared senior authorship.

Cite this article: Kaidesoja M, Aaltonen S, Bogl LH, Heikkilä K, Kaartinen S, Kujala UM, Kärkkäinen U, Masip G, Mustelin L, Palviainen T, Pietiläinen KH, Rottensteiner M, Sipilä PN, Rose RJ, Keski-Rahkonen A, and Kaprio J. (2019) FinnTwin16: A Longitudinal Study from Age 16 of a Population-Based Finnish Twin Cohort. Twin Research and Human Genetics 22: 530-539, https://doi.org/10.1017/ thg.2019.106 addition to use/abuse of alcohol and its long-term consequences have been smoking, physical activity, overall physical health, eating behaviors and eating disorders, weight development, obesity, life satisfaction and personality. This review focuses on research areas that have been a focus since 2010, but does not comprehensively review every study and publication that has been completely or partially based on the cohort data. In addition to continued follow-up of alcohol exposure, these key research areas include physical activity, eating behaviors, eating disorders, weight development and obesity.

\section{Studying Health Behaviors in Adolescents and Young Adults (Waves 1-4)}

Driven by the goal to better understand the developmental course of health behaviors from midadolescence into adulthood, we initiated the FT16 study in 1991 with a baseline assessment of five consecutive birth cohorts of 16-year-old twins born during 1974-1979 and ascertained from the Central Population Register of Finland. For the first round of data collection, we contacted 3215 twin pairs with both co-twins alive, resident in Finland at the time of baseline assessment and living with one or both parents (see Table 1 for more information). For the

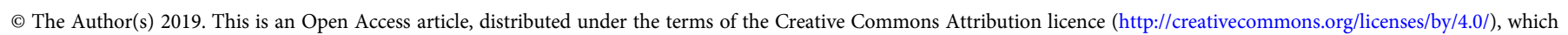
permits unrestricted re-use, distribution, and reproduction in any medium, provided the original work is properly cited. 


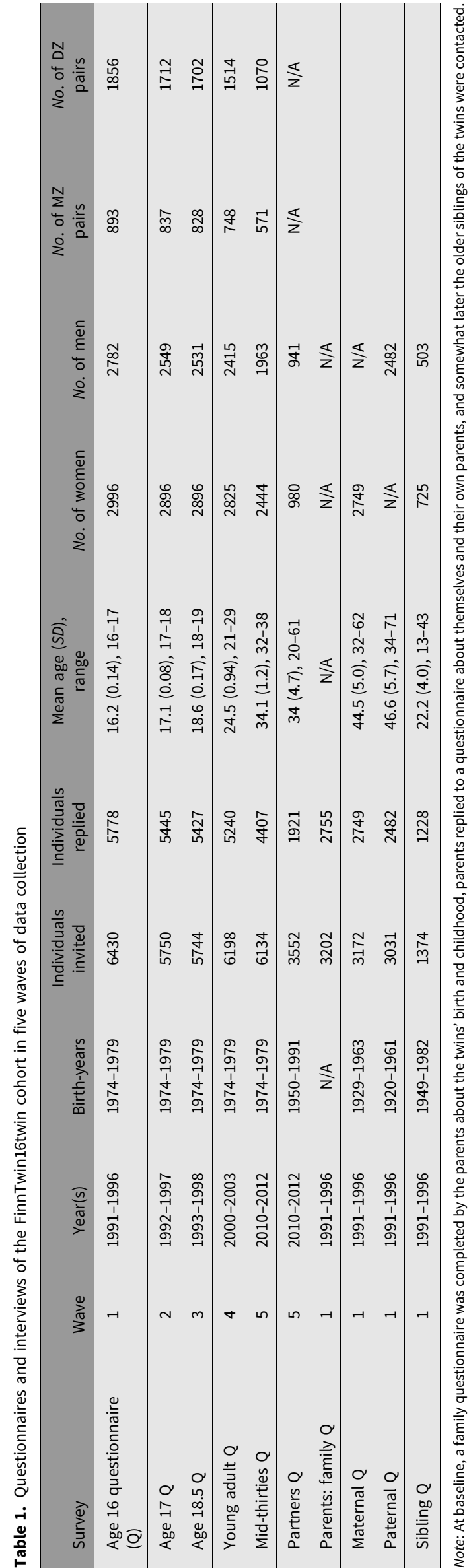

Table 2. Number of families informative for transgenerational and longitudinal analyses

\begin{tabular}{lccccc}
\hline Family definition & $\begin{array}{c}\text { All } \\
\text { pairs }\end{array}$ & $\begin{array}{c}\text { MZ } \\
\text { pairs }\end{array}$ & $\begin{array}{c}\text { SSDZ } \\
\text { pairs }\end{array}$ & $\begin{array}{c}\text { OSDZ } \\
\text { pairs }\end{array}$ & $\begin{array}{c}\text { XZ } \\
\text { pairs }\end{array}$ \\
\hline Both parents replied at baseline & 1969 & 624 & 638 & 657 & 50 \\
\hline $\begin{array}{l}\text { At least one parent replied } \\
\text { at baseline }\end{array}$ & 2351 & 754 & 759 & 779 & 59 \\
\hline $\begin{array}{l}\text { At least one parent and at least } \\
\text { one sibling replied at baseline }\end{array}$ & 807 & 240 & 276 & 271 & 20 \\
\hline
\end{tabular}

Note: Inclusion criteria are that the twins have replied to at least one of the three adolescent questionnaires and to at least one of the two adult questionnaires. This provides longitudinal data while adding parents with or without an older sibling provides transgenerational information. $\mathrm{MZ}=$ monozygotic twin pairs; $\mathrm{SSDZ}=$ same-sex dizygotic twin pairs; $\mathrm{OSDZ}=$ oppositesex dizygotic twin pairs; $\mathrm{XZ}=$ twin pairs with uncertain zygosity.

representativeness of the data, we also included the Swedishspeaking minority (around $5.2 \%$ of the Finnish population in 2018; Official Statistics of Finland, 2019) in the study, with all questionnaires in the mother tongue of the participant. Ten waves of the baseline questionnaire were sent each of the 5 years, timed to twins' birthdays, to achieve rigorous age standardization. The twins' parents were sent individual questionnaires at baseline, and one older sibling was included in some families (see Table 2). The twins completed the second- and third-wave questionnaires when they were 17 , timed to the twins' birthdays, and 18.5 years old, within a 3-month target of this mean age, respectively. We administered the fourth-wave questionnaire when the twins were young adults, telescoping the data collections for each birth year to a 6-month period during 2000-2002 (age 24 on average; Kaprio, 2006), but with some questionnaires being returned in early 2003.

The items in the first questionnaire focused on health habits, including alcohol consumption and attitudes, tobacco use, physical health, physical activity and eating habits. The twins' personalities and relationships with their co-twins were assessed. The parents completed a family questionnaire on the twins' birth, infancy and childhood experiences. The parents and some older siblings also completed individual questionnaires about their own health habits, substance use, lifestyle and personality. Zygosity was determined using the questionnaire method on similarity of appearance in childhood and confusion by strangers, with use of complementary items supplied by the parents. The procedure and first distributions are reported by Kaprio et al. (1995). Later, we have confirmed zygosity using genetic marker sets in 603 pairs to date.

For each wave, the number of twins genotyped or with methylation data is listed in Table 3; for the most part, DNA samples have been collected in later clinical subsamples, and hence are not contemporaneous with the major study surveys.

As research interests expanded and new areas relevant to the twins' life stage emerged, we added new research topics to the fourth-wave questionnaire. In addition to the topics covered in previous rounds, the fourth-wave questionnaire included items related to overall life satisfaction and mental well-being, romantic relationships and sexuality, place of residence, oral health and eating disorders. The eating disorder items aimed to identify symptoms of disordered eating, and based on these items, a selected group of participants was screened for eating disorders (for more details, see Keski-Rahkonen, Hoek et al., 2007; KeskiRahkonen et al., 2006). Data on alcohol consumption, physical 
Table 3. Number of deaths and the numbers genotyped or with methylation data, and the number of pairs genotyped on genome-wide array

\begin{tabular}{lccccc}
\hline Survey & $\begin{array}{c}\text { N of deaths } \\
\text { by } 1 / 1 / 2018\end{array}$ & $\begin{array}{c}\text { N with } \\
\text { genotype data }\end{array}$ & $\begin{array}{c}\text { N with } \\
\text { methylation data }\end{array}$ & $\begin{array}{c}\text { N of MZ pairs with at least } \\
\text { one twin genotyped }\end{array}$ & $\begin{array}{c}N \text { of DZ pairs with both } \\
\text { twins genotyped }\end{array}$ \\
\hline Age 16 Q & 86 & 1228 & 305 & 290 & 321 \\
\hline Age 17 Q & 80 & 1200 & 299 & 283 & 312 \\
\hline Age 18.5 Q & 78 & 1207 & 302 & 280 & 317 \\
\hline Young adult Q & 50 & 1215 & 302 & 277 & 318 \\
\hline Mid-thirties Q & 17 & 1071 & 278 & 236 & 241 \\
\hline
\end{tabular}

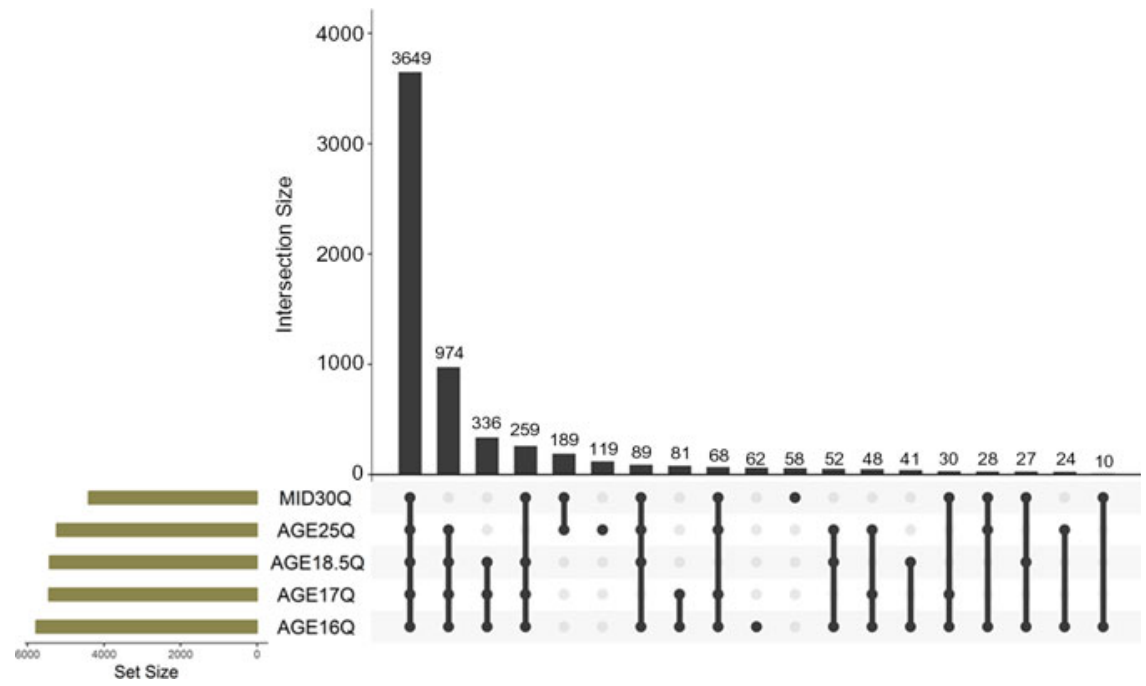

Fig. 1. The combinations of responses to the questionnaires.

activity, overall physical health, weight, eating habits, religiosity and studies/work were expanded with new, more detailed items. We also asked the twins to measure their waist circumference with a measuring tape sent alongside the questionnaire.

For a more detailed description of the first four waves of the FT16 study, see Kaprio (2006, 2013) and Kaprio et al. (2002).

To study metabolism and obesity in more detail, the TwinFat substudy was initiated during the fourth wave. It included twins from the FinnTwin12 cohort (FT12; Kaprio et al., 2002) and the FT16 cohort. The twins were chosen based on their body mass index (BMI), with the aim to cover the full BMI range of both normal weight and obese participants and a full range of withinpair differences in BMI. For more information, see Bogl et al. (2016) and below.

\section{Studying Health Behaviors in Twins in Their Thirties (Wave 5)}

To expand the valuable longitudinal dataset already gathered, the fifth wave of the FT16 study included many of the same research topics as the previous rounds. New supplements were reading disabilities, handedness and physical activity-related motivation. In addition, personality was reassessed with a different measure than in the baseline assessment. It was also important to include topics related to the life stage of the twins now in their thirties, and thus we inquired about romantic relationships, offspring and fertility intentions. The twins' partners were also included in the fifth wave.
Another new supplement was the administration of an internet-based battery of neuropsychological tests on spatial ability, memory and reading speed to selected participants. Some of the partners were asked to administer the same battery of neuropsychological tests.

The data collection of the fifth wave was initiated in May 2010 with a pilot study and continued October 2010-November 2011 for Finnish-speaking subjects and spring of 2012 for Swedishspeaking subjects. The twins were now aged 32-38 (mean age at response 34.1 years). As opposed to the previous waves conducted with mailed questionnaires, the fifth wave was conducted using internet-based questionnaires.

The cohort has over 3000 twin pairs, and the invitation for the fifth round of data collection was sent to all twins living in Finland irrespective of previous participation, excluding the ones who had previously declined participation. This was done to update all cohort members of their inclusion in the cohort as adolescents and to permit them to withdraw from the study if they chose to do so. Of the 6134 twins that were contacted, 4407 responded, which gave a response rate of $71.9 \%$. Combinations of responses from the participants to questionnaires in all the five waves can be seen in Figure 1. For the neuropsychological tests, we received responses from 1391 participants.

The partners of the twins completed their internet-based questionnaire using a fully anonymous code, supplied in a closed envelope to the twin to be given onward to the partner. Altogether, 1949 of the partners responded and spouse pair responses (i.e., both twin and spouse responded) were obtained from 1826 dyads. 
Results for the neuropsychological tests were obtained from 710 partners and 677 spouse pair dyads.

To study how physical activity level is associated with body composition, glucose homeostasis and brain morphology in young adult male monozygotic (MZ) twin pairs discordant for physical activity, the FITFATTWIN substudy was initiated during the fifth wave. For more information, see Rottensteiner et al. (2015).

\section{Measures of Overall Health}

The items related to health covered the respondent's selfassessments of health, self-reported symptoms and diseases, use of medicine and sick leave.

Self-rated health was assessed with one item ('How would you describe your current state of health?') with five response alternatives that allowed the respondent to estimate their health from 'Very good' to 'Poor.' Self-related health in the previous waves of this cohort is further elaborated in Silventoinen et al. (2007).

The questionnaire also included questions addressing musculoskeletal pain that were modified from a questionnaire developed by Mikkelsson et al. (1997). The items inquired whether and how often the respondent had had pain or aches during the previous months in the neck-shoulder region, lower back and stomach. In addition, the questionnaire inquired about headache, problems falling asleep or waking up and nervousness.

Backache symptoms were surveyed in three ways, including frequency of lower back pain, number of backache episodes lasting over 1 day and the type of backache at worst. These items were based on questions used in our study of back pain in the older cohort (Battié et al., 2007).

In addition, we asked a medical history of 12 different chronic conditions.

Eating disorders were addressed with a separate item ('Has anyone ever thought you might suffer from an eating disorder?').

We also asked about other chronic physical conditions that impact daily activities, illness-related absences from work and about regular medications.

The survey presented to the twins' partners included three items related to overall health: one asking for a self-assessment of their current state of health, one on possible long-term disease affecting their everyday functioning and one on the quantity of illnessrelated absences from work during the past 12 months.

Reading disabilities were screened with three items originally developed for the Jyväskylä Longitudinal Study of Dyslexia (JLD) and shown to be effective in screening for reading and writing disabilities (Lyytinen et al., 2015). These included asking the respondent whether they had ever had problems in reading or writing, whether they learned to read early or late in relation to their peers and whether anyone in their family had had problems in reading or writing. These items were also included in the partners' questionnaire. These items have been used to screen participants for a more detailed study of dyslexiadiscordant and concordant twin pairs as part of the JLD study.

\section{Measures of Leisure-Time Physical Activity and Motivation for Physical Activity}

The assessment of leisure-time physical activity level was based on a series of structured questions on leisure-time physical activity behavior. The twins reported the frequency, mean duration and mean intensity of their leisure-time physical activity sessions as well as their physical activity during journeys to and from work.
All types of leisure-time and commuting-related physical activity were taken into account. The items can be used separately, or the overall activity level can be assessed based on the items by transforming the items into leisure-time metabolic equivalent units (METs). The leisure-time METs are created by assigning a multiple of the resting metabolic rate to each activity, and by calculating the product of the activity, defined as intensity $\times$ duration $\times$ frequency. The overall activity levels are expressed as the sum score of leisure-time MET hours/day.

The twins also gave a self-assessment of their current physical fitness by choosing from a 5-point Likert scale. In addition, they reported the type of sports they participate in on a regular basis by answering a multiple-choice question that consists of 26 different sports and an open field with space for reporting up to three additional sports. We also inquired about how physically demanding their work or studies are, and the daily duration of physically demanding/physically not demanding hobbies and housework.

Motives for leisure-time physical activity were assessed using a modified version of the validated Recreational Exercise Motivation Measure (REMM; Aaltonen et al., 2017; Aaltonen et al., 2014; Pajunen, 2004; Rogers \& Morris, 2003). The REMM was initially designed to assess adults' physical activity motives, and it is based on self-determination theory (Deci \& Ryan, 1985). The original version of the REMM consists of 73 items representing both intrinsic and extrinsic motives for leisure-time physical activity. These items form eight dimensions of 8-13 items. In the FT16 study, each of the eight dimensions was represented by only one item as follows: (1) mastery, (2) enjoyment, (3) affiliation, (4) competition/ego, (5) others' expectations, (6) physical fitness, (7) psychological state and (8) appearance. The motive dimensions of 'mastery' and 'enjoyment' represent intrinsic motives, while 'affiliation,' 'competition/ego,' 'others' expectations,' 'physical fitness,' 'psychological state' and 'appearance' represent extrinsic motives. Both the original REMM (Rogers \& Morris, 2003) and the Finnish version of the 73-item REMM (Pajunen, 2004) have been validated.

\section{Measures of Alcohol and Tobacco Consumption}

In the fifth wave, as in all previous waves, patterns of alcohol consumption were assessed extensively. The frequency of drinking alcohol was assessed with the same item included in all earlier FT16 questionnaires ('How often do you consume alcohol?'); the nine response options ranged from 'Daily' to 'I do not consume alcohol.' Another item addressed frequency of high-density drinking (more than five bottles of beer or a similar amount of alcohol within one occasion), with 10 response options ranging from 'Daily' to 'I do not consume alcohol.' A similar item addressed the frequency of intoxication, asking 'How often do you get drunk?' We also asked about the frequency of alcohol consumption during the past 4 weeks and typical quantity of alcohol consumption on the days in which alcohol was used. One item addressed the maximum quantity ever drunk in a 24 -h period (further elaborated in relation to eating disorders in Mustelin et al., 2016).

To address problems with alcohol, we used an 11-item modified version of the Malmö-modified Michigan Alcoholism Screening test (MmMAST; Kaprio et al., 2002; Selzer 1971; Seppä et al., 1992) and the Rutgers Alcohol Problem Index (RAPI; Dick et al., 2011; White \& Labouvie, 1989), which addresses self-reported alcohol-related problems experienced in the past 12 months. For 
this study, an adapted 22-item version of RAPI was used because the item related to school and homework was excluded. Both the MmMAST and the RAPI had been included in the questionnaire administered at age 24, and the RAPI also at age 18 .

Use of illicit drugs was assessed as follows: 'Have you ever used hashish, marijuana or other drugs, or for example sniffed glue?' The five response options ranged from 'Never' to '20 times or more.'

We also asked for current smoking status, use of pipe, cigars or smokeless tobacco (snus) and experience with illegal drugs (e.g., cannabis). Smokers completed the Fagerstrom Test for Cigarette Dependence (Fagerström, 2012).

The twins' partners answered three items related to alcohol consumption, covering the frequency and magnitude of their alcohol consumption. We also asked for their current smoking status.

\section{Measures of Eating Habits}

Diet quality was assessed with a short food frequency questionnaire (FFQ) that included questions on the consumption frequency of 11 different food and beverage items. Another question inquired about the slices of bread consumed per day separately for dark bread, mixed flour bread and white bread. The questionnaire was formulated according to current Nordic (Nordic Council of Ministers, 2012) and Finnish nutrition recommendations (National Nutrition Council, 2014) and modified from a short FFQ that was previously administered to Finnish adults (Leppälä et al., 2010). Based on the short FFQ, we developed a diet quality score (DQS) where a higher score indicates a better diet quality. Our study of a subsample from the cohort showed that a higher DQS was associated with better diet quality, as indicated by positive correlations with dietary intakes of protein, fiber and magnesium, and negative correlations with intakes of total fat, saturated fat and sucrose (Masip et al., 2019).

We also addressed eating styles, including questions on meal frequency, the regularity of eating, health-conscious eating, night eating, external and emotional eating, and snacking behaviors with a questionnaire previously developed for this purpose (Keski-Rahkonen, Bulik et al., 2007). Eating styles were assessed by asking the respondent to choose from four different options the one that best described their eating style.

\section{Measures of Psychological Wellbeing, Life Satisfaction and Personality}

To address current level of psychological distress, we administered the 12-item version of the General Health Questionnaire (GHQ; Goldberg, 1972). The GHQ was included also in the partners' questionnaire, and had been asked in the fourth wave as well.

We assessed life satisfaction with the widely used five-item Satisfaction with Life Scale (SWLS; Diener et al., 1985), which has a 7-point Likert-style response scale. In addition, we further assessed life satisfaction by asking the respondent to rate their satisfaction with their free time at home and outside home, and their work and their relationship with their twin on a 5-point Likert scale. The SWLS was included also in the partners' questionnaire.

The 15-item GSOEP Big Five Inventory (BFI-S; Gerlitz \& Schupp, 2005) provided an assessment of personality. The partner of the twin assessed their own personality and their partner's personality with the original 44-item version of the BFI (John et al., 1991). The baseline assessment of personality of the 16-year-old twins was done using selected scales from the Minnesota Multiphasic Personality Inventory (MMPI; Hathaway \& McKinley, 1940).

\section{Measures Related to Romantic Relationships, Offspring and Fertility Intentions}

Two items addressed romantic relationships by inquiring about the number of relationships with cohabiting and the duration of a possible current relationship.

Possible offspring was addressed by asking whether the respondent had any biological children or whether she or his partner was currently pregnant. The items also addressed any nonbiological children, miscarriages or abortions. The twins reporting no offspring answered items about possible attempts to get pregnant and the timing of these attempts.

If the respondent reported having children, they also answered more detailed items about the offspring. These included the child's/children's gender, age and handedness. The items also addressed time to pregnancy and whether the pregnancy was planned (items from the British National Child Development Study; Power \& Elliot, 2006). We also asked about breastfeeding and whether the respondent's current partner was the biological mother/father of the child/children.

Fertility intentions were addressed by two items inquiring about the desired number of children and the self-assessed probability of having more children.

The same measures were presented in the partners' questionnaire, adding two items about the quantity of time spent with their partner and their level of satisfaction with different aspects of the relationship.

\section{Other Measures}

The twins answered two items designed to determine their handedness (Heikkilä et al., 2015).

They also reported their current weight and height. In addition, they were asked more detailed questions related to changes in their weight (heaviest and lowest weight in current height). They also reported weight losses of over $5 \mathrm{~kg}$ or over $10 \mathrm{~kg}$ and their self-perceived ideal weight. The partner's survey included the same items related to weight and height, excluding the items concerning changes in weight and weight losses of over $10 \mathrm{~kg}$.

As in the fourth wave, the twins were provided with a tape measure and instructions to measure their waist circumference (Mustelin et al., 2009).

The questionnaire also included items inquiring about the current living and financial situation of the twin and items related to their work and studies. We also inquired whether they still lived with their twin and how frequently they kept contact with their twin.

\section{Studies Based on the FinnTwin16 Data Since 2010}

\section{Leisure-Time Physical Activity}

FT16 data have been used to understand several physical activityrelated domains. With our longitudinal dataset, we showed the existence of age-specific genetic and environmental influences on physical activity: heritability estimates for physical activity varied between $31 \%$ and $42 \%$ from adolescence to adulthood (Aaltonen et al., 2013). In a study comparing the motives for physical activity among twins who had been consistently active and consistently inactive over time, we noticed clear differences 
in motives for physical activity between the physical activity levels (Aaltonen et al., 2014). Interestingly, the motives for physical activity themselves were also revealed to be influenced by genetic factors (Aaltonen et al., 2017).

In an analysis of the diversity of physical activity, we found that participation in a higher number of sport activities in adolescence predicted a higher level of leisure-time physical activity in adulthood, although this may be partly attributed to common familial factors (Mäkelä et al., 2017). Another ongoing study found that participation in several different sports was associated with less low back pain but not with less neck-shoulder region pain in adult twins. Yet, participation in several sports in adolescence did not predict either low back pain or neck-shoulder region pain in adulthood.

Our study on the association of leisure-time physical activity level on waist circumference showed that an increase in the level of leisure-time physical activity or remaining active was associated with lower waist gain over the almost 10 -year follow-up period. Decreasing leisure-time physical activity level, irrespective of the baseline activity level, led to waist gain resembling that of the persistently inactive participants (Rottensteiner et al., 2014). Our results suggest that changes in leisure-time physical activity level are associated with changes in easily measured waist circumference already among young adults.

We also found the number of physical activities/sports engaged in to be inversely associated with waist circumference, also when adjusted for overall leisure-time physical activity level volume (Rottensteiner et al., 2017). This indicates that engaging in a diversity of physical activities/sports rather than specific activities may be beneficial in preventing abdominal obesity.

Furthermore, in-depth clinical measurements of health-related factors such as fitness, body composition, glucose homeostasis and brain morphology were conducted for the FITFATTWIN subsample of twins discordant for leisure-time physical activity level. In this clinical substudy, healthy male twins were studied to see whether differences arising from differing physical activity levels are observable prior to the onset of overt chronic diseases or to the presence of widespread abnormal values for other cardiometabolic risk factors (Rottensteiner et al., 2015, 2016). Systematically selected 10 male MZ twin pairs discordant for leisure-time physical activity habits over the past 3 years also showed a significant difference in cardiorespiratory fitness measured by a maximal exercise test (spiroergometry). In the absence of any overall BMI difference $(\sim 3 \%)$, the less active co-twins tended to have more body fat $(\sim 21 \%)$ measured by dual energy $\mathrm{x}$-ray absorptiometry (DEXA), and had an average $31 \%$ more intra-abdominal adipose tissue measured by magnetic resonance imaging (MRI), and $41 \%$ more intraperitoneal adipose tissue (IAAT) compared with their genetically identical but more active twin brothers. Diet did not differ between the co-twins (Rottensteiner et al., 2016). The active twin brothers had a lower homeostatic model assessment (HOMA) index and higher insulin sensitivity index (Matsuda index, calculated from glucose and insulin values from an oral glucose tolerance test), indicating better glucose homeostasis (Rottensteiner et al., 2015). Our results demonstrated that the factors known to be related to reduced cardiometabolic risk are already associated in healthy men at a young age with the level of leisure-time physical activity level. Regional volumetric analyses of the whole brain MRI revealed that striatal and prefrontal cortex (subgyral and inferior frontal gyrus) brain gray matter volumes were larger in the nondominant hemisphere in active twins compared with less active co-twins
(Rottensteiner et al., 2015), which indicates site-specific responses in the brain to improve motor control.

The pooled dataset of the FT12 and FT16 cohorts was used in a study that investigated whether within-pair differences in motor development in childhood as reported by parents predict within-pair differences in leisure-time physical activity level as reported by twins themselves in later life (Aaltonen et al., 2015). The study found that having been more advanced in motor development skills as a child predicted higher leisure-time physical activity levels in adulthood, at least partly independent of family background in both genders.

\section{Obesity and Metabolism}

In the TwinFat substudy, we have taken advantage of the MZ BMI-discordant twin design to study the effects of acquired obesity. In this design, all phenotypic differences within pairs are by definition acquired (i.e., due to lifestyle), as the heavier and leaner co-twins are inherently matched for genetic background, age, sex and shared family environment. MZ twin pairs who are discordant for obesity are extremely rare and represent only about $2 \%$ of the twin population.

To better understand the consequences of BMI-discordance in MZ twins, we have examined 25 BMI-discordant MZ pairs (within-pair difference in $\mathrm{BMI} \geq 3 \mathrm{~kg} / \mathrm{m}^{2}$ and weight difference $>10 \mathrm{~kg}$ ) in our extensive metabolic studies. The twins completed an intensive protocol, consisting of adipose tissue, muscle and skin biopsies, and blood sampling during a 2 -h oral glucose tolerance test (OGTT; substudy with a meal test), urine and feces samples, extensive examination of anthropometrics, body composition (DEXA), subcutaneous and visceral fat content (MRI), intrahepatic and intramyocellular fat (MR spectroscopy), calorimetry, assessment of behavioral traits (questionnaires and interviews on past and current food intake, food preferences, physical activity, use of alcohol and smoking, health-related attitudes, weight history, family history and quality of life, food diaries).

In the BMI-discordant $\mathrm{MZ}$ twins, we have demonstrated that metabolism is heavily influenced by acquired obesity. The heavier co-twins weighed on average $18 \mathrm{~kg}$ more, had $28 \%$ more total fat, $67 \%$ more subcutaneous fat, $200 \%$ more intra-abdominal fat and $300 \%$ more liver fat than their leaner counterparts (Heinonen et al., 2015, 2017; Jukarainen et al., 2016; Kaye et al., 2017; Naukkarinen et al., 2014; Pietiläinen et al., 2016; Rämö et al., 2017). Consequently, the heavier co-twins had significantly decreased insulin sensitivity (Naukkarinen et al., 2014), hyperinsulinemia, blunted gut hormone (glucagon-like peptide-1) response to glucose and meals (Matikainen et al., 2013), as well as derangements in circulating lipoproteins (Kaye et al., 2013; Pietiläinen et al., 2009), coagulation factors (Kaye et al., 2012), proteomics (Sahebekhtiari et al., 2019) and metabolomics profiles (Bogl et al., 2016; Rämö et al., 2017). Our epigenomic analyses indicate significantly differentially methylated genomic areas in the blood (Ollikainen et al., 2015) and subcutaneous adipose tissue (Pietiläinen et al., 2016) of co-twins with obesity compared with their lean co-twins, with robust associations with metabolic disturbances.

The main novel finding from the MZ BMI-discordant twin studies has been that a key process associated with the development of metabolic derangements in obesity is mitochondrial downregulation in the adipose tissue (Heinonen et al., 2015; Naukkarinen et al., 2014, Muniandy et al., 2017) and adipocytes (Heinonen et al., 2017); in particular, a reduction in the 
nicotinamide adenine dinucleotide $(\mathrm{NAD}+)$ metabolism (Jukarainen et al., 2016). Downregulation of mitochondrial biogenesis paralleled poor adipocyte differentiation, increased fat cell size, increased adipose tissue and systemic inflammation, and high liver fat (Heinonen et al., 2014; Muniandy et al., 2017; Naukkarinen et al., 2014). As mitochondria are needed for healthy expansion of the adipose tissue, our results strongly suggest that mitochondrial downregulation leads to an inability of subcutaneous adipose cells to store lipids, and an overflow of free fatty acids to organs that normally do not accumulate fat (such as ectopic lipid accumulation into the liver). This, in turn, leads to impairment of whole-body metabolism, as fatty liver is a central driver of glucose intolerance, dyslipidemia, coagulation disorders, inflammation and other features of the metabolic syndrome (Kaye et al., 2013; Naukkarinen et al., 2014; Rämö et al., 2017).

In summary, the studies using the BMI-discordant $\mathrm{MZ}$ twin model show that adipose tissue dysfunction, especially downregulation of the mitochondrial pathways, is intricately linked to the development of metabolic complications in obesity. The results obtained in this project propose that mitochondrial flexibility to adapt to overnutrition, to dissipate and store excess energy safely and to drive proliferation of healthy new fat cells in the adipose tissue is key in the prevention of ectopic fat deposition, circulating nutrient overload and metabolic disturbances.

\section{Weight-Related Behaviors}

To explore weight-related behaviors and their long-term health consequences in the general population, the twins were studied as individuals by adjusting correlated observations within twin pairs. Overall, weight-related ideals and behaviors appeared to have significant associations not only with weight change but also with physical and mental health in young adults over a period of 10 years (waves 4-5; Kärkkäinen et al., 2016, 2018a, 2018b). Most young adults gained weight over 10 years. Only about one out of every four young adults were able to successfully maintain his/her weight during this 10-year period (Kärkkäinen et al., 2018b).

The majority of young women and almost every other young man were dissatisfied with their weight at age 24 (Kärkkäinen et al., 2016). However, for most young adults, weight ideals at age 24 did not influence future weight change (Kärkkäinen et al., 2016). With respect to the weight-related behaviors, two factors were significant to predict future weight maintenance in both sexes: regular eating and not trying to lose weight (Kärkkäinen et al., 2018b). Moreover, disordered eating behaviors at age 25 predicted long-term health-related consequences, particularly psychological distress at age 34 (Kärkkäinen et al., 2018a).

\section{Eating Disorders}

We also used data from this cohort to study the outcomes and correlates of eating disorders. As eating disorder diagnoses were made at wave 4 , the sample was restricted to the subjects who participated at wave 4 . The screening and diagnostic interviews for eating disorders are described in detail elsewhere (KeskiRahkonen et al., 2006; Keski-Rahkonen, Hoek et al., 2007).

In a study on anorexia nervosa we found that its long-term prognosis might be better than previously thought (Mustelin, Raevuori, Bulik et al., 2015). The majority of previous studies on the topic were conducted in clinical populations and indicated a poor prognosis for a substantial proportion of the individuals.
Yet, in this community-based sample, the long-term prognosis of anorexia nervosa appeared promising: weight restoration was common and sociodemographic outcomes were generally favorable, although women with a history of anorexia nervosa were less likely than their peers to have children.

In a longitudinal study on risky drinking behaviors among women with eating disorders, women with a history of anorexia nervosa and bulimia nervosa scored higher than their peers on measures of alcohol-related problems in their thirties (Mustelin et al., 2016). We have also studied body weight trajectories of individuals with binge eating disorder (Mustelin, Raevuori, Hoek et al., 2015) and related behaviors (Mustelin et al., 2017). We found that these individuals were heavier than their peers throughout follow-up and that the differences persisted into their thirties.

\section{Outcomes Associated With Adolescent Alcohol Exposure and Parental Alcohol Abuse}

Several analyses of FT16 data have evaluated short- and long-term associations of adolescent alcohol exposure. Within-family comparisons of drinking-discordant co-twins have been a powerful design for asking whether such associations are consequences of familial confounds or, potentially, causal in nature. An initial study (Dick et al., 2011) asked how well the RAPI, administered in late adolescence, predicts alcohol diagnosis over a 7-year follow-up. Age 18 RAPI scores correlated (.55) with symptom counts of alcohol use disorder (AUD) at age 25. Within-family comparisons of 117 twin pairs discordant for both age 18 RAPI and age 25 AUD diagnoses replicated between-family associations of twins as individuals, suggesting that one pathway to problem drinking in early adulthood is a direct one from problem drinking in adolescence.

Neurocognitive effects of adolescent alcohol abuse at age 25 were found in components of auditory event-related potentials (ERP) among FT16 twin pairs selected for pairwise discordance/ concordance for their age 18 RAPI scores (Koskinen et al., 2011). Intrapair differences in ERPs were associated with intrapair differences in alcohol variables, consistent with the inference that adolescent alcohol abuse is associated with subtle neurophysiological changes in attention and orienting.

A third study of drinking-discordant FT16 twin pairs (Rose et al., 2014) associated RAPI scores at 18 with a diverse set of behavioral outcomes at age 25: the adverse outcomes included sustained substance abuse, poor health, physical symptoms, early coital debut, multiple sexual partners, life dissatisfaction, truncated education and financial problems in early adulthood. The within-family comparisons yielded more compelling evidence than previously available that adolescent alcohol exposure disrupts transitions into early adulthood.

A fourth study of FT16 pairs discordant for alcohol use disorder (Ruggeri et al., 2015) included a genome-wide analysis of DNA methylation in 18 discordant MZ pairs identified from intrapair differences in their age 18 and age 24 RAPI scores and the differences in AUD symptoms found in face-to-face interviews at age 25. Concurrent with the interviews, blood was obtained to confirm zygosity and for extraction of DNA. Hypermethylation in the PPMIG gene locus was associated with AUD, and these regions were characterized in a sample of $\sim 500$ nontwin adolescents who associated PPMIG hypermethylation with early escalation of alcohol use and increased behavioral impulsivity - first evidence for an epigenetic marker associated with elevated 
adolescent drinking and, inferentially, its underlying neurobehavioral expression. With three twin pairs, we have since piloted an MRI protocol for a planned proposal to make structural and functional comparisons of brain imaging in $\mathrm{MZ}$ pairs from FT16 who were consistently discordant for alcohol exposure from adolescence to age 40-45.

In addition, Pyry Sipilä is studying the association of paternal and maternal problem drinking with lifetime problem drinking of their adult offspring. The parents of the twins reported on their problem drinking (measured with MmMAST) when the twins were aged 16, and the twins reported on an 11-item lifetime version of the same measure when aged 25 and 34 . In the study, paternal problem drinking was a modest predictor of lifetime problem drinking of their adult children. Maternal problem drinking showed similar but less statistically robust associations.

\section{Future Directions}

As detailed above, the FT16 cohort has provided an ample data resource and contributed widely to the study of health-related behaviors. Future waves of data collection will reassess research topics detailed above, but will also include new research areas related to the life stage of the participants. Our current plan is to initiate wave 6 by conducting an online survey comparable to the wave 5, with a focus on key variables and measures that we have studied multiple times. In addition, we need to consider that as these twins are approaching midlife, their personal, family and occupational circumstances change. Also, they are entering an age period in which metabolic and subclinical indicators of future somatic disease are more common or more severe. Thus, our study design and study domains need to reflect their circumstances. Studying individuals across their life course will offer an extensive view of how health behaviors and their consequences unfold.

Financial support. Data collection and analyses have been supported by the National Institute of Alcohol Abuse and Alcoholism (R.J.R., grant numbers AA-12502, AA-00145 and AA-09203); the Academy of Finland (J.K., grant numbers 100499, 205585, 118555, 141054, 264146, 308248 and 312073), (K.H.P, grant numbers 272376, 266286, 314383 and 315035), (S.A., grant number 266592); the Sigrid Juselius Foundation (J.K. and K.H.P.); Finnish Medical Foundation (K.H.P.); Finnish Diabetes Research Foundation (K.H.P.); Novo Nordisk Foundation (K.H.P.), Gyllenberg Foundation (K.H.P. and U.K.); Helsinki University Hospital Research Funds (K.H.P.); Government Research Funds (K.H.P.); University of Helsinki (K.H.P.); Juho Vainio Foundation (U.M.K., U.K., S.A. and G.M.); META-PREDICT (U.M.K., within the European Union Seventh Framework Programme, HEALTH-F2-2012-277936); the Finnish Ministry of Education and Culture (S.A. and U.M.K.); the Finnish Cultural Foundation (S.A. and U.K.), (G.M., grant number 00180705); AA Turunen Foundation (U.K.); DocPop in Population Health (U.K.); The Finnish Foundation for Alcohol Studies (P.N.S.) and Helsinki Institute of Life Science (P.N.S.).

Conflicts of interest. None.

\section{References}

Aaltonen, S., Kaprio, J., Vuoksimaa, E., Huppertz, C., Kujala, U. M., \& Silventoinen, K. (2017). Genetic architecture of motives for leisure-time physical activity: A twin study. Scandinavian Journal of Medicine \& Science in Sports, 27, 1431-1441.

Aaltonen, S., Latvala, A., Rose, R., Pulkkinen, L., Kujala, U., Kaprio, J., \& Silventoinen, K. (2015). Motor development and physical activity: A longitudinal discordant twin-pair study. Medicine \& Science in Sports \& Exercise, 47, 2111-2118.
Aaltonen, S., Ortega-Alonso, A., Kujala, U. M., \& Kaprio, J. (2013). Genetic and environmental influences on longitudinal changes in leisure-time physical activity from adolescence to young adulthood. Twin Research and Human Genetics, 16, 535-543.

Aaltonen, S., Rottensteiner, M., Kaprio, J., \& Kujala, U. M. (2014). Motives for physical activity among active and inactive persons in their mid-30s. Scandinavian Journal of Medicine \& Science in Sports, 24, 727-735.

Battié, M. C., Videman, T., Levalahti, E., Gill, K., \& Kaprio, J. (2007). Heritability of low back pain and the role of disc degeneration. Pain, 131, 272-280

Bogl, L. H., Kaye, S. M., Rämö, J. T., Kangas, A. J., Soininen, P., Hakkarainen, A., . . Pietiläinen, K. H. (2016). Abdominal obesity and circulating metabolites: A twin study approach. Metabolism, 65, 111-121.

Deci, E. L., \& Ryan, R. M. (1985). Intrinsic motivation and self-determination in human behavior. New York, NY: Plenum.

Dick, D. M., Aliev, F., Viken, R., Kaprio, J., \& Rose, R. J. (2011). Rutgers alcohol problem index scores at age 18 predict alcohol dependence diagnoses 7 years later. Alcoholism: Clinical and Experimental Research, 35, 1011-1014.

Diener, E., Emmons, R. A., Larsen, R. J., \& Griffin, S. (1985). The satisfaction with life scale. Journal of Personality Assessment, 49, 71-75.

Fagerström, K. (2012). Determinants of tobacco use and renaming the FTND to the Fagerström test for cigarette dependence. Nicotine \& Tobacco Research, 14, 75-78.

Gerlitz, Y., \& Schupp, J. (2005). Zur Erhebung der Big-Five-basierten Persönlichkeitsmerkmale im SOEP [Assessment of Big Five personality characteristics in the SOEP]. German Institute of Economic Research (Research Notes 4). Berlin, Germany: DIW.

Goldberg, D. (1972). The detection of psychiatric illness by questionnaire: A technique for the identification and assessment of non-psychotic psychiatric illness. London, UK: Oxford University Press.

Hathaway, S. R., \& McKinley, J. C. (1940). A multiphasic personality schedule (Minnesota): 1. Construction of the schedule. Journal of Psychology, 10, 249-254.

Heikkilä, K., Vuoksimaa, E., Saari-Kemppainen, A., Kaprio, J., Rose, R. J., Haukka, J., ... Iivanainen, M. (2015). Higher prevalence of lefthandedness in twins? Not after controlling birth time confounders. Twin Research and Human Genetics, 18, 526-532.

Heinonen, S., Buzkova, J., Muniandy, M., Kaksonen, R., Ollikainen, M., Ismail, K., . . Pietiläinen, K. H. (2015) Impaired mitochondrial biogenesis in adipose tissue in acquired obesity. Diabetes, 64, 3135-3145.

Heinonen, S., Muniandy, M., Buzkova, J., Mardinoglu, A., Rodríguez, A., Frühbeck, G., _. Pietiläinen, K. H. (2017). Mitochondria-related transcriptional signature is downregulated in adipocytes in obesity: a study of young healthy MZ twins. Diabetologia, 60, 169-181.

Heinonen, S., Saarinen, L., Naukkarinen, J., Rodríguez, A., Frühbeck, G., Hakkarainen, A., ... Pietiläinen, K. H. (2014). Adipocyte morphology and implications for metabolic derangements in acquired obesity. International Journal of Obesity, 38, 1423-1431.

John, O. P., Donahue, E. M., \& Kentle, K. L. (1991). The Big Five Inventory Versions 44 and 54. Berkeley, CA: University of California at Berkeley, Institute of Personality and Social Research.

Jukarainen, S., Heinonen, S., Rämö, J. T., Rinnankoski-Tuikka, R., Rappou, E., Tummers, M., . . P Pietiläinen, K. H. (2016). Obesity is associated with low NAD+/SIRT pathway expression in adipose tissue of BMI-discordant monozygotic twins. Journal of Clinical Endocrinology \& Metabolism, 101, 275-283.

Kaprio, J. (2006). Twin studies in Finland 2006. Twin Research and Human Genetics, 9, 772-777.

Kaprio, J. (2013). The Finnish twin cohort study: An update. Twin Research and Human Genetics, 16, 157-162.

Kaprio, J., Pulkkinen, L., \& Rose, R. J. (2002). Genetic and environmental factors. Twin Research, 5, 366-371.

Kaprio, J., Rimpelä, A., Winter, T., Viken, R. J., Rimpelä, M., \& Rose, R.J. (1995). Common genetic influences on BMI and age at menarche. Human Biology, 67, 739-753. 
Kärkkäinen, U., Mustelin, L., Raevuori, A., Kaprio, J., \& Keski-Rahkonen, A. (2016). Ideals versus reality: Are weight ideals associated with weight change in the population? Obesity, 24, 947-953.

Kärkkäinen, U., Mustelin, L., Raevuori, A., Kaprio, J., \& Keski-Rahkonen, A. (2018a). Do disordered eating behaviours have long-term health-related consequences? European Eating Disorders Review, 26, 22-28.

Kärkkäinen, U., Mustelin, L., Raevuori, A., Kaprio, J., \& Keski-Rahkonen, A. (2018b). Successful weight maintainers among young adults - A ten-year prospective population study. Eating Behaviors, 29, 91-98.

Kaye, S., Lokki, A. I., Hanttu, A., Nissilä, E., Heinonen, S., Hakkarainen, A., Pietiläinen, K. H. (2017). Upregulation of early and downregulation of terminal pathway complement genes in subcutaneous adipose tissue and adipocytes in acquired obesity. Frontiers in Immunology, 8, 545.

Kaye, S. M., Maranghi, M., Bogl, L. H., Kaprio, J., Hakkarainen, A., Lundbom, J., ... Pietiläinen, K. H. (2013). Acquired liver fat is a key determinant of serum lipid alterations in healthy monozygotic twins. Obesity, 21, $1815-1822$.

Kaye, S. M., Pietiläinen, K. H., Kotronen, A., Joutsi-Korhonen, L., Kaprio, J., Yki-Järvinen, H., . . Rissanen, A. (2012). Obesity-related derangements of coagulation and fibrinolysis: A study of obesity-discordant monozygotic twin pairs. Obesity, 20, 88-94.

Keski-Rahkonen, A., Bulik, C. M., Pietiläinen, K. H., Rose, R. J., Kaprio, J., \& Rissanen, A. (2007). Eating styles, overweight and obesity in young adult twins. European Journal of Clinical Nutrition, 61, 822-829.

Keski-Rahkonen, A., Hoek, H. W., Susser, E.Z., Linna, M.S, Sihvola, E., Raevuori, A., . . Rissanen, A. (2007). Epidemiology and course of anorexia nervosa in the community. American Journal of Psychiatry, 164, 1259-1265.

Keski-Rahkonen, A., Sihvola, E., Raevuori, A., Kaukoranta, J., Bulik, C.M., Hoek, H.W., . . Kaprio, J. (2006). Reliability of self-reported eating disorders: Optimizing population screening. International Journal of Eating Disorders, 39, 754-762.

Koskinen, S. M., Ahveninen, J., Kujala, T., Kaprio, J., O'Donnell, B.F., Osipova, D., ... Rose, R. J. (2011). A longitudinal twin study of adolescent alcohol abuse on the neurophysiology of attention and orienting. A longitudinal twin study of effects of adolescent alcohol abuse on the neurophysiology of attention and orienting. Alcohol: Clinical \& Experimental Research, $35,1339-1350$.

Leppälä, J., Lagström, H., Kaljonen, A., \& Laitinen, K. (2010). Construction and evaluation of a self-contained index for assessment of diet quality. Scandinavian Journal of Public Health, 38, 794-802.

Lyytinen, H., Erskine, J., Hämäläinen, J., Torppa, M., \& Ronimus, M. (2015). Dyslexia - Early identification and prevention: Highlights from the Jyväskylä Longitudinal Study of Dyslexia. Current Developmental Disorders Reports, 2, 330-338.

Mäkelä, S., Aaltonen, S., Korhonen, T., Rose, R. J., \& Kaprio, J. (2017). Diversity of leisure-time sport activities in adolescence as a predictor of leisure-time physical activity in adulthood. Scandinavian Journal of Medicine \& Science in Sports, 27, 1902-1912.

Masip, G., Keski-Rahkonen, A., Pietiläinen, K. H., Kujala, U. M., Rottensteiner, M., Väisänen, K., . . Bogl, L. H. (2019). Development of a food-based diet quality score and associations with eating styles and nutrient intakes in Finnish twins. Nutrients, 11 pii: E2561. doi: 10.3390/ nu11112561. PubMed PMID: 31652865.

Matikainen, N., Bogl, L. H., Hakkarainen, A., Lundbom, J., Lundbom, N., Kaprio, J., . . Pietiläinen, K. H. (2013). GLP-1 responses are heritable and blunted in acquired obesity with high liver fat and insulin resistance. Diabetes Care, 37, 242-251.

Mikkelsson, M., Salminen, J. J., \& Kautiainen, H. (1997). Non-specific musculoskeletal pain in preadolescents prevalence and 1-year persistence. Pain, 73, 29-35.

Muniandy, M., Heinonen, S., Yki-Järvinen, H., Hakkarainen, A., Lundbom, J., Lundbom, N., . . Pietiläinen, K. H. (2017). Gene expression profile of subcutaneous adipose tissue in BMI-discordant monozygotic twin pairs unravels molecular and clinical changes associated with sub-types of obesity. International Journal of Obesity, 41, 1176-1184.

Mustelin, L., Bulik, C. M., Kaprio, J., \& Keski-Rahkonen, A. (2017). Prevalence and correlates of binge eating disorder related features in the community. Appetite, 109, 165-171.
Mustelin, L., Latvala, A., Raevuori, A., Rose, R. J., Kaprio, J., \& KeskiRahkonen, A. (2016). Risky drinking behaviors among women with eating disorders - A longitudinal community-based study. International Journal of Eating Disorders, 49, 563-571.

Mustelin, L., Raevuori, A., Bulik, C. M., Rissanen, A., Hoek, H. W., Kaprio, J., \& Keski-Rahkonen, A. (2015). Long-term outcome in anorexia nervosa in the community. International Journal of Eating Disorders, 48, 851-859.

Mustelin, L., Raevuori, A., Hoek, H. W., Kaprio, J., \& Keski-Rahkonen, A. (2015). Incidence and weight trajectories of binge eating disorder among young women in the community. International Journal of Eating Disorders, $48,1106-1112$

Mustelin, L., Silventoinen, K., Pietiläinen, K., Rissanen, A., \& Kaprio, J. (2009). Physical activity reduces the influence of genetic effects on BMI and waist circumference: A study in young adult twins. International Journal of Obesity, 33, 29-36.

National Nutrition Council. (2014). Finnish nutrition recommendations Health from food [Suomalaiset ravitsemussuositukset - Terveyttä ruoasta]. Helsinki: Juvenes Print.

Naukkarinen, J., Heinonen, S., Hakkarainen, A., Lundbom, J., Vuolteenaho, K., Saarinen, L., . . . Pietiläinen, K. (2014). Characterizing metabolically healthy obesity in weight-discordant monozygotic twins. Diabetologia, 57, 167-176.

Nordic Council of Ministers. (2012). Nordic Nutrition Recommendations: Integrating nutrition and physical activity (5th ed.). Copenhagen, Denmark: Nordisk Ministerråd.

Official Statistics of Finland (OSF) (2019). Population structure. Helsinki: Statistics Finland. Retrieved from http://www.stat.fi/til/vaerak/index_en.html

Ollikainen, M., Ismail, K., Gervin, K., Kyllönen, A., Hakkarainen, A., Lundbom, J., ... Kaprio, J. (2015). Genome-wide blood DNA methylation alterations at regulatory elements and heterochromatic regions in monozygotic twins discordant for obesity and liver fat. Clinical Epigenetics, 7, 39.

Pajunen, K. (2004). Vapaa-ajan liikunnan motivaatiomittarin psykometriset ominaisuudet (Unpublished master's thesis). University of Jyväskylä, Jyväskylä, Finland.

Pietiläinen, K. H., Ismail, K., Järvinen, E., Heinonen, S., Tummers, M., Bollepalli, S., ... Ollikainen, M. (2016). DNA methylation and gene expression patterns in adipose tissue differ significantly within young adult monozygotic BMI-discordant twin pairs. International Journal of Obesity, 40, 654-661.

Pietiläinen, K. H., Söderlund, S., Rissanen, A., Nakanishi, S., Jauhiainen, M., Taskinen, M., \& Kaprio, J. (2009). HDL subspecies in young adult twins: Heritability and impact of overweight. Obesity, 17, 1208-1214.

Power, C., \& Elliott, J. (2006). Cohort profile: 1958 British birth cohort (National Child Development Study). International Journal of Epidemiology, 35, 34-41.

Rämö, J., Kaye, S., Jukarainen, S., Bogl, L., Hakkarainen, A., Lundbom, J., . Pietiläinen, K. (2017). Liver fat and insulin sensitivity define metabolite profiles during a glucose tolerance test in young adult twins. Journal of Clinical Endocrinology \& Metabolism, 102, 220-231.

Rogers, H., \& Morris, T. (2003). An overview of the development and validation of the Recreational Exercise Motivation Measure (REMM). In R. Stelter (Ed.), New approaches to exercise and sport psychology: Theories, methods and applications. Proceedings of the 11th European Congress of Sport Psychology [CD-ROM]. Copenhagen, Denmark: University of Copenhagen.

Rose, R. J., Winter, T. M., Vikjen, R. J., \& Kaprio, J. (2014). Adolescent alcohol abuse and adverse adult outcomes: Evaluating confounds with drinking-discordant twins. Alcoholism: Clinical and Experimental Research, 38, 2314-2321.

Rottensteiner, M., Leskinen, T., Järvelä-Reijonen, E., Väisänen, K., Aaltonen, S., Kaprio, J., \& Kujala, U. M. (2016). Leisure-time physical activity and intra-abdominal fat in young adulthood: A monozygotic co-twin control study. Obesity, 24, 1185-1191.

Rottensteiner, M., Leskinen, T., Niskanen, E., Aaltonen, S., Mutikainen, S., Wikgren, J., ... Kujala, U. (2015). Physical activity, fitness, glucose homeostasis, and brain morphology in twins. Medicine \& Science in Sports \& Exercise, 47, 509-518.

Rottensteiner, M., Mäkelä, S., Bogl, L. H., Törmäkangas, T., Kaprio, J., \& Kujala, U. M. (2017). Sport disciplines, types of sports, and waist circumference in young adulthood - A population-based twin study. European Journal of Sport Science, 17, 1184-1193. 
Rottensteiner, M., Pietiläinen, K. H., Kaprio, J., \& Kujala, U. M. (2014). Persistence or change in leisure-time physical activity habits and waist gain during early adulthood: A twin-study. Obesity, 22, 2061-2070.

Ruggeri, B., Nymberg, C., Vuoksimaa, E., Lourdusamy, A., Wong, C.P., Carvalho, F. M., ... IMAGEN Consortium. (2015). Association of protein phosphatase PPMIG with alcohol use disorder and brain activity during behavioral control in a genome-wide methylation analysis. American Journal of Psychiatry, 172, 543-552.

Sahebekhtiari, N., Saraswat, M., Joenväärä, S., Jokinen, R., Lovric, A., Kaye, S., ... Pietiläinen, K. (2019). Plasma proteomics analysis reveals dysregulation of complement proteins and inflammation in acquired obesity - A study on rare BMI-discordant monozygotic twin pairs. Proteomics - Clinical Applications, 13, e1800173.
Selzer, M. L. (1971). The Michigan Alcoholism Screening Test: The quest for a new diagnostic instrument. American Journal of Psychiatry, 127, 1653-1658.

Seppä, K., Koivula, T., \& Sillanaukee, P. (1992). Drinking habits and detection of heavy drinking among middle-aged women. Addiction, 87, 1703-1709.

Silventoinen, K., Posthuma, D., Lahelma, E., Rose, R. J., \& Kaprio, J. (2007). Genetic and environmental factors affecting self-rated health from age 16-25: A longitudinal study of Finnish twins. Behavioral Genetics, 37, $326-333$.

White, H. R., \& Labouvie, E. W. (1989). Towards the assessment of adolescent problem drinking. Journal of Studies on Alcohol, 50, 30-37. 